\title{
LIPOID LIVER DISEASE AND STEATITIS IN A CAPTIVE SAPPHIRE DAMSEL, POMACENTRUS PAVO
}

\author{
Jaime L. WEISMAN, Debra L. MILLER* \\ Veterinary Diagnostic and Investigational Laboratory, The University of Georgia, \\ College of Veterinary Medicine, Tifton, GA, USA
}

\begin{abstract}
Weisman J.L., Miller D.L. 2006. Lipoid liver disease and steatitis in a captive sapphire damsel, Pomacentrus pavo. Acta Ichthyol. Piscat. 36 (2): 99-104.
\end{abstract}

\begin{abstract}
Background. Sapphire damselfish, Pomacentrus pavo (Bloch, 1787) (family Pomacentridae) are reef fish found in tropical and temperate oceans but also are popular aquaria species in commercial and private operations; yet, there remains a paucity of information regarding diseases in this species. Thus, reporting of disease occurrences in this species is vital and serves as a foundation upon which to gather and build our knowledge base for this species.

Material and Methods. A captive sapphire damsel presented with severe nonresolving abdominal distension. The fish was humanely euthanized and fixed tissues submitted for routine histological examination. Additionally, special histochemical stains were done including: periodic acid-Schiff's (PAS), modified acid-fast, Pearl's iron, and Gomori methenamine silver (GMS).

Results. Grossly, a brown gelatinous material was coating the coelomic cavity and its contents, resulting in adhesion of the coelomic organs. The liver was diffusely pale. Histological examination revealed severe hepatocellular vacuolation and sheets of adipocytes replacing the hepatic parenchyma. Positive staining was observed in hepatocytes and pigmented macrophages with modified acid-fast, Pearl's iron, and periodic acid Schiff's. Inflammatory cell infiltrates were multifocally scattered throughout the abdominal fat and mesentery.

Conclusion. The gross and histological findings were consistent with lipoid liver disease (ceroidosis), steatitis, and peritonitis. Possible contributing factors are vitamin E deficiency/depletion, rancid feed, and chronic stress. This case represents an initial documentation for this disease in this species.
\end{abstract}

Keywords: fish, sapphire damsel, blue damselfish, Pomacentrus pavo, ceroidosis, lipoid liver disease, steatitis

\section{INTRODUCTION}

The sapphire damsel, Pomacentrus pavo (Bloch, 1787) (family Pomacentridae) (known also as the blue damselfish) is a small (maximum of ca. $8 \mathrm{~cm}$ for free-ranging specimens) fish found in tropical and temperate waters through the world. They are one of the most popular aquaria species in commercial and private operations and are one of the top ten most traded species which together account for approximately $36 \%$ of the marine fish traded (Wabnitz et al. 2003). Besides the neurofibromatosis-like disease reported in bicolor damselfish, (Schmale and Hensley 1988, Campbell et al. 2001, Schmale et al. 2002) reports on other diseases affecting damselfish are lacking. Thus, it is important to document diseases as they occur in this species so that we might use this information to better manage them in captivity and perhaps better understand their needs in the wild.
Lipoid liver disease is a serious and often fatal disease of captive fish that has primarily been studied in rainbow trout fisheries (Holliman and Southgate 1986). It also has been described in the common dab (Begg et al. 2000) and an African stonefish, (Penrith et al. 1994). Sequelaes to lipoid liver disease typically include anemia and immune suppression that may lead to secondary bacterial infections (Penrith et al. 1994, Hardy 2001). Herein we present a case of lipoid liver disease in a sapphire damsel.

\section{MATERIALS AND METHODS}

A live adult male sapphire damsel from a private collection was examined in March 2006 with a 5 month history of progressive abdominal distension. The fish had been part of the collection for 4 years and was housed in a 100-L upright hexagonal aquarium along with a yellow tang, Zebrasoma flavescens (Bennett, 1828). The fishes 
were fed commercially available dry food, along with thawed frozen brine shrimp, 1-2 times weekly. Water temperature and salinity were maintained at ca. $25^{\circ} \mathrm{C}$ and $1.025 \mathrm{ppm}$, respectively. The damselfish usually measure ca. $5 \mathrm{~cm}$ in total length, $1.5 \mathrm{~cm}$ height (at pectoral fins), and are 8-mm thick (at level of pectoral fins), but the specimen examined, was approximately two times its normal body size. Additionally, the fish had a dark and dull skin color, extended fins and scales, and tended to remain at the surface and gulp air. The fish was humanely euthanized, using $250 \mathrm{mg} \cdot \mathrm{L}^{-1}$ benzocaine hydrochloride, and subjected to a necropsy. Other than the kidney and heart, the coelomic tissues were adhered to one another by a gelatinous substance and not easily separated without causing collection artifact that may have interfered with histological examination. Therefore the adhered tissues along with the remaining cadaver were placed in $10 \%$ buffered formalin and submitted for further processing.

Fixed tissues were routinely processed, paraffin embedded, sectioned, placed on glass slides, stained with hematoxylin and eosin, cover slipped, and viewed by light microscopy. Tissue sections also were stained with periodic acid-Schiff's (PAS), modified acid-fast, Pearl's iron, Gomori methenamine silver (GMS), acid-fast and LillieTwort, and examined with light microscopy. These special stains were chosen to identify polysaccharides, ceroid, hemosiderin, fungal agents, acid-fast organisms, and Gram positive or negative bacteria, respectively. Additionally, similarly processed liver from an apparently normal captive blue damsel fish of unknown sex but similar age served as a control for special stains.

\section{RESULTS}

Grossly, the lining of the coelomic cavity was brown to tan and contained a brown gelatinous material (presumed to be fat) surrounding and adhering the coelomic organs. The adhered coelomic organs were difficult to separate (Fig. 1). The liver was diffusely pale.

Histological examination revealed sheets of adipocytes replacing the hepatic parenchyma and lipid (presumptive) degeneration within the remaining hepatocytes (Fig. 2). Pigmented macrophages were scattered throughout the liver, mesentery, and occasionally other organs. Hepatocytes and pigmented macrophages stained positive with modified acid-fast and PAS stain, and variably positive for Pearl's iron (Fig. 3). Additionally, melanomacrophage centers often displayed positive staining with Pearl's iron (Fig. 3D). Acid-fast, Lillie-Twort and GMS were negative for acid-fast organisms, bacteria and fungi, respectively. The abdominal fat and mesentery had coalescing to

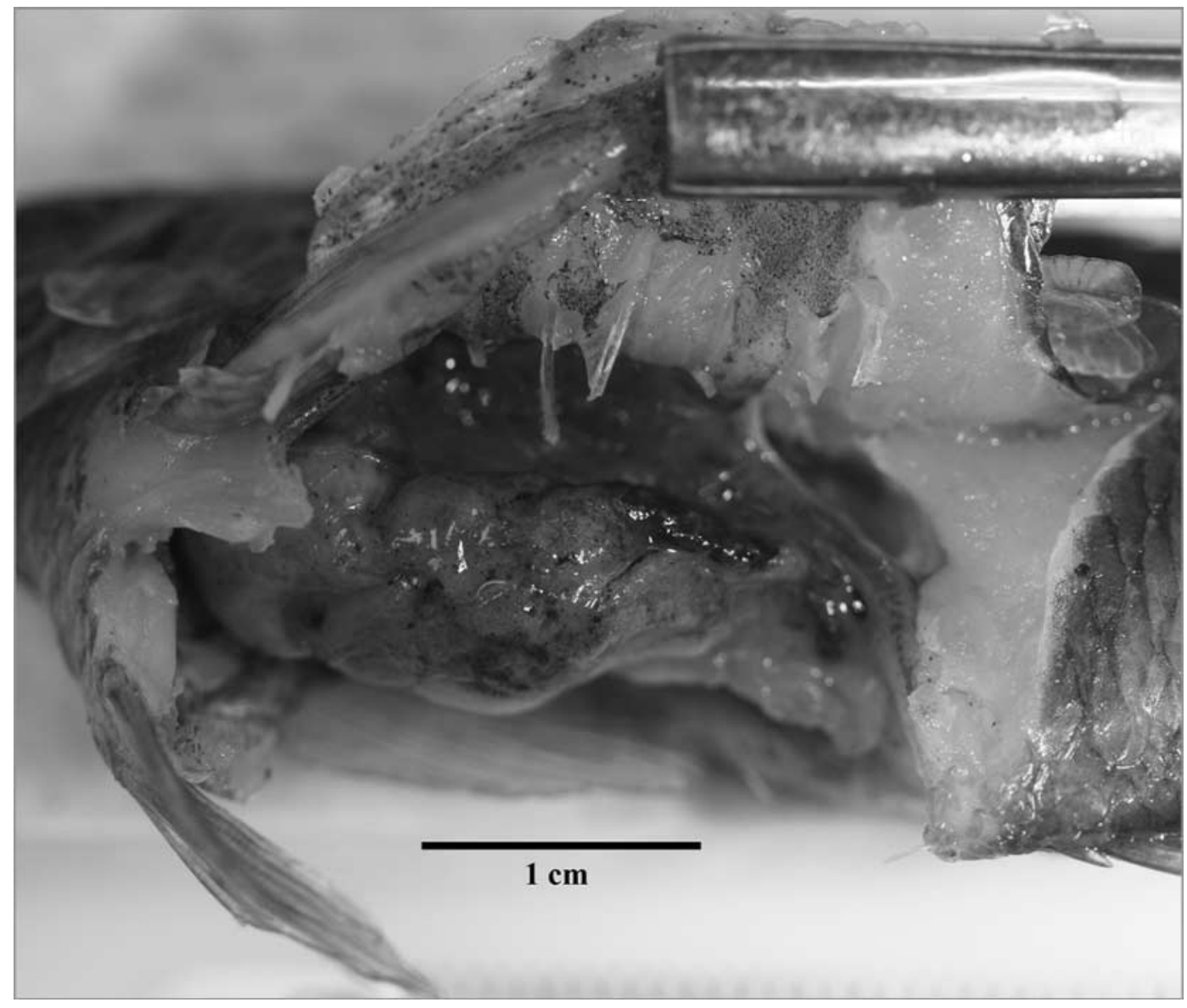

Fig. 1. Macroscopic view of the coelomic cavity of a sapphire damsel, Pomacentrus pavo, presented with nonresolvable abdominal distension; the coelomic organs were adhered to one another by a fine, gelatinous, clear to brown material 


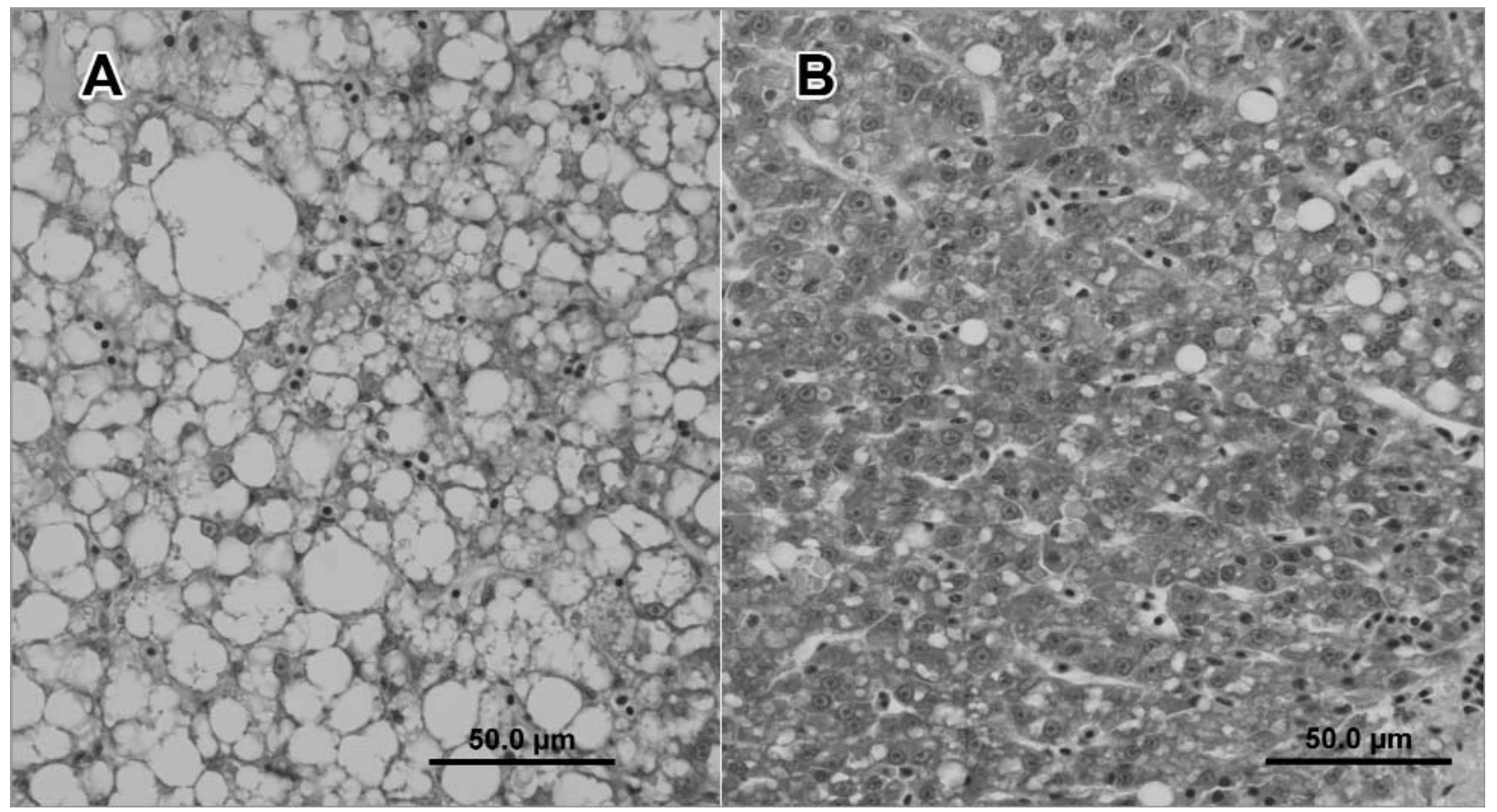

Fig. 2. Microscopic views of livers from 2 sapphire damsel, Pomacentrus pavo; (A) liver of the damselfish with lipoid liver disease; (B) liver from an apparently normal damselfish shown for comparison

diffuse infiltrates of inflammatory cells (including macrophages, lymphocytes, neutrophilic granulocytes, and scattered eosinophilic granulocytes), fine bands of fibrosis, multifocal pale amorphous eosinophilic matrix (edema), and fat necrosis (Fig. 4A). The serosal surfaces of the internal organs showed minimal to mild expansion by mixed inflammatory cells. Rarely, myocyte degeneration with cytoplasmic vacuolation, loss of cross striations, fragmentation, and infiltration by mild numbers of inflammatory cells was noted (Fig. 4B). Scattered granulomas were present throughout the liver and coelomic cavity. The granulomas were often mature with thick concentric bands of fibrous connective tissue and occasionally were surrounded by aggregates of macrophages containing goldenbrown pigment. No other significant changes were noted.

\section{DISCUSSION}

Both the gross and light microscopic changes seen in this case are consistent with lipoid liver disease (ceroidosis), steatitis and peritonitis (serositis and mesenteritis). Granulomas are a common response to numerous insults in fish, including bacterial infections, parasite migration, and foreign- or otherwise irritating substances (such as leakage of secretory material). Further, granulomas have been associated with steatitis in fish (Herman and Kircheis 1985). In this damselfish, the granulomas were mostly advanced, displaying concentric fibrosis suggesting past insults and therefore, it remains unknown if they developed around ceroid/iron containing macrophages or were due to past infections, parasites or foreign substances. Similarly, melanomacrophages are a common finding in most fishes, but those staining positive for ceroid or iron in this fish were possibly the result of the lipoid liver disease.
Lipoid liver disease is diagnosed histologically by the presence of hepatic lipoid infiltration, the presence of ceroid-containing macrophages, myocyte degeneration, and hemolysis manifested as increased iron deposits in hepatocytes and pigmented macrophages (Hardy 2001). The latter effect (hemolysis) results from the toxic effect of lipid auto-oxidation products (free radicals, peroxide aldehydes and ketones) on the renal hematopoietic tissues and erythrocytes (Hardy 2001). These histological changes were consistent with what was observed in the damselfish in this case, excepting that renal hematopoietic necrosis was not seen. Presentation of this disease may vary depending on stage of the disease and contributing factors (e.g., type of auto-oxidation product produced) to the disease (Tacon 1996, Holliman and Southgate 1986, Hardy 2001). Lipoid liver disease, steatitis and myocyte degeneration may be attributed to vitamin E deficiency, such as may occur following ingestion of rancid feed (Smith 1979, Holliman and Southgate 1986, Tacon 1996). Other possible factors leading to vitamin E deficiency in ocean dwelling organisms may include chronic stress or other oxidative stress (Sakai et al. 1998, Berntssen et al. 2003, Welker and Congleton 2004, Goodwin 2006). Vitamin E is an antioxidant that helps prevent peroxidation of adipose tissue (Comporti 1993). In rainbow trout, it has been shown that increasing the amount of $\alpha$-tocopherol (vitamin E) in the diet can prevent lipoid liver disease, demonstrating that proper diet is essential to prevent this disease (Smith 1979). Although fresh (thawed frozen) food items were part of the normal husbandry regime of the damselfish in this case presentation, vitamin E supplementation was not. Further, failure to remove uneaten thawed frozen food or improper storage of frozen food may have resulted in ran- 


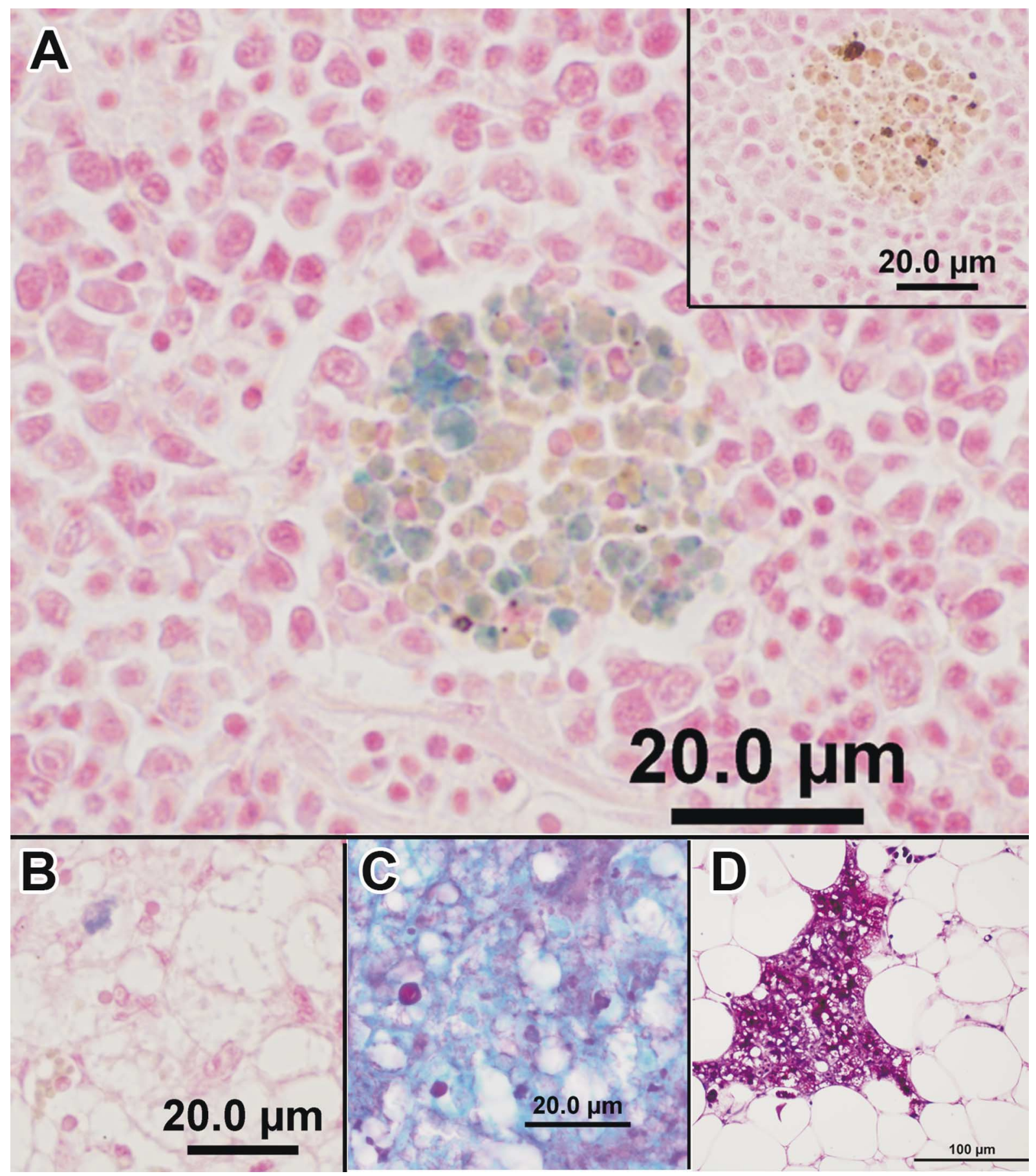

Fig. 3. Microscopic views of special staining of tissues from a sapphire damsel, Pomacentrus pavo, with lipoid liver disease; (A) Pearl's iron staining of a melanomacrophage center in the spleen; inset shows lack of similar staining in a melanomacrophage center from the spleen of an apparently normal damselfish for comparison; positive staining for Pearl's iron (B), periodic acid Schiff's (C), and modified acid-fast (D) was observed in the liver of the fish with lipoid liver disease

cidity, which could have led to vitamin E deficiency. We suggest that vitamin E deficiency may have served as the etiology for the lipoid liver disease and steatitis in this damselfish.

\section{CONCLUSION}

This case demonstrates that lipoid liver disease and steatitis can affect sapphire damsel and should be considered in cases of abdominal distension in captive animals.
This serves as information that can be added to the paucity of data currently available for this popular reef fish. Understanding the diseases and nutritional needs of this and other reef fish is vital to better understand the needs of both captive individuals and free-ranging species. Further, better management of captive individuals will decrease the demand for additional collection of free-ranging individuals and hopefully aid in the conservation of reef species. 


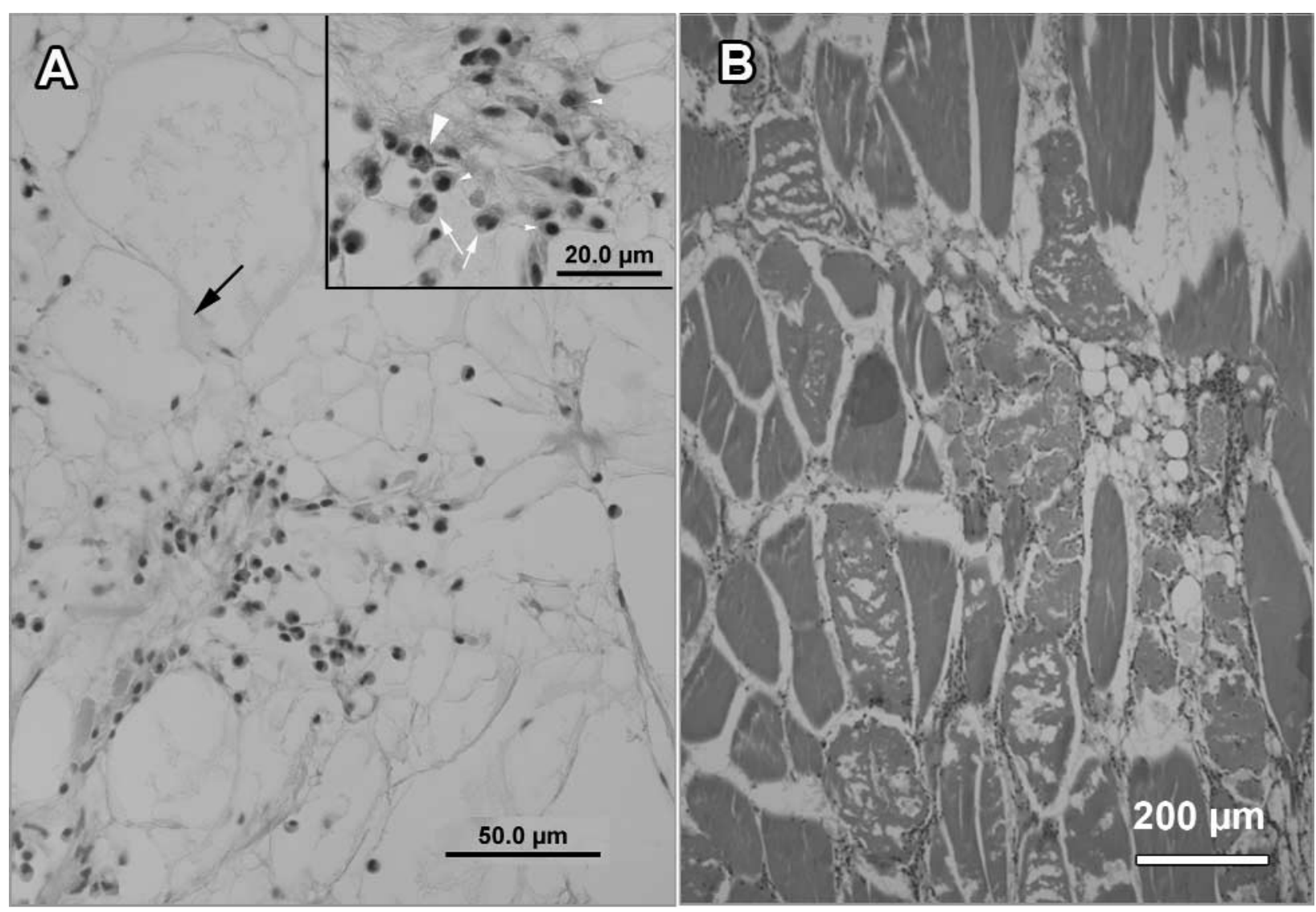

Fig. 4. Microscopic view of tissues from a sapphire damsel, Pomacentrus pavo, with lipoid liver disease; (A) abdominal fat with infiltrates of mixed inflammatory cells and pale amorphous eosinophilic matrix (edema, black arrow); inset show higher power view of neutrophilic granulocytes (arrows), eosinophilic granulocyte (large arrowhead) and mononuclear (macrophages and lymphocytes) cells (small arrowheads); (B) myocyte degeneration and necrosis

\section{ACKNOWLEDGEMENTS}

We would like to thank Dr. Matthew Gray for donation of this fish. We acknowledge the staff of the UGA Tifton VDIL for their help in tissue processing.

\section{REFERENCES}

Begg G.S., Bruno D.W., McVicar A.H. 2000. The histopathology and ultrastructure of steatitis affecting common dab Limanda limanda. Diseases of Aquatic Organisms 41: 123-133.

Berntssen M.H.G., Aatland A., Handy R.D. 2003. Chronic dietary mercury exposure causes oxidative stress, brain lesions, and altered behaviour in Atlantic salmon (Salmo salar) parr. Aquatic Toxicology 65: 55-72.

Campbell C.E., Gibbs P.D.L., Schmale M.C. 2001. Progression of infection and tumor development in damselfish. Marine Biotechnology (NY) 3 (Suppl. 1): 107-114.

Comporti M. 1993. Lipid peroxidation. Biopathological significance. Molecular Aspects of Medicine 14: 199-207.

Goodwin A.E. 2006. Steatitis, fin loss and skin ulcers of channel catfish, Ictalurus punctatus (Rafinesque), fin- gerlings fed salmonid diets. Journal of Fish Diseases 29: 61-64.

Hardy R.W. 2001. The nutritional pathology of teleosts. Pp. 347-365. In: Roberts R.J. (ed.) Fish Pathology, 3rd edn. W.B. Saunders, London, Edinburgh, New York, Philadelphia, St. Louis, Sydney, Toronto.

Herman R.L., Kircheis F.W. 1985. Steatitis in Sunapee trout, Salvelinus alpinus oquassa Girard. Journal of Fish Diseases 8: 237-239.

Holliman A., Southgate P. 1986. Serious losses in rainbow trout (Salmo gairdneri) associated with hepatocellular ceroidosis. The Veterinary Record 119: 179.

Penrith M.L., Bastianello S.S., Penrith M.J. 1994. Hepatic lipoidosis and fatty infiltration of organs in a captive African stonefish, Synanceja verrucosa Bloch and Schneider. Journal of Fish Diseases 17: 171-176.

Sakai T., Murata H., Endo M., Shimomura T., Yamauchi K., Ito T., Yamaguchi T., Nakajima H., Fukudome M. 1998. Severe oxidative stress is thought to be a principal cause of jaundice of yellowtail, Seriola quinqueradiata. Aquaculture 160: 205-214.

Smith C.E. 2002. A virus-like agent associated with neurofibromatosis in damselfish. Diseases of Aquatic Organisms 49: 107-115. 
Schmale M.C., Hensley G.T. 1988. Transmissibility of Wabnitz C.T., Taylor M., Green E., Razak T. 2003. a neurofibromatosis-like disease in bicolor damselfish. Cancer Research 48: 3828-3833.

Smith C.E. 1979. The prevention of liver lipoid degeneration (ceroidosis) and microcytic anaemia in rainbow trout Salmo gairdneri Richardson fed rancid diets: a preliminary report. Journal of Fish Diseases 2: 429437.

Tacon A.G.C. 1996. Lipid nutritional pathology in farmed fish. Archiv für Tierernahrung - Archives of Animal Nutrition 49: 33-39.

From ocean to aquarium: The global trade in marine ornamental species. UNEP-WCMC Biodiversity Series, Cambridge, UK.

Welker T.L., Congleton J.L. 2004. Oxidative stress in juvenile Chinook salmon, Oncorhynchus tshawytscha (Walbaum). Aquaculture Research 35: 881-887.

Received: 23 August 2006

Accepted: 28 November 2006

Published electronically: 31 December 2006 